\title{
Reverse Takotsubo cardiomyopathy with left bundle branch block after anesthesia induction in a patient with subarachnoid hemorrhage: a case report
}

\author{
Eun Kyung Choi ${ }^{1}$, Jong-Hoon $\mathrm{Kim}^{2}$, Minhyun Kim ${ }^{1}$ \\ ${ }^{1}$ Department of Anesthesiology and Pain Medicine, Yeungnam University College of Medicine, Daegu, Korea \\ ${ }^{2}$ Department of Neurosurgery, Yeungnam University College of Medicine, Daegu, Korea
}

\begin{abstract}
Takotsubo or reverse Takotsubo cardiomyopathy is a well-known cardiac complication of subarachnoid hemorrhage (SAH) that shows transient left ventricular wall motion abnormalities with electrocardiogram (ECG) changes. ST change followed by T inversion is a common ECG finding complicated with these disorders, left bundle branch block (LBBB) may be a potential ECG pattern which is seen. In this case, we describe the clinical profile and outcomes of a patient with LBBB and reverse Takotsubo cardiomyopathy after anesthetic induction, which was scheduled as an emergent external ventricular drainage after SAH. This is the first report of an LBBB pattern in reverse Takotsubo cardiomyopathy.
\end{abstract}

Keywords: Anesthesia; Bundle-branch block; Electrocardiography; Subarachnoid hemorrhage; Takotsubo cardiomyopathy

\section{Introduction}

Takotsubo cardiomyopathy is a well-known cardiac complication of subarachnoid hemorrhage (SAH). This disorder shows transient left ventricular wall motion abnormalities with electrocardiogram (ECG) changes in the absence of coronary artery disease [1]. Reverse Takotsubo cardiomyopathy is a variant of Takotsubo cardiomyopathy that presents a little different characteristic profile [2]. Takotsubo cardiomyopathy is characterized by transient left ventricular dysfunction with hypocontractile apex and compensatory hypercontractile base. On the other hand, reverse Takotsubo cardiomyopathy shows the opposite left ventricular movement, that is, a hypokinetic basal ventricular segment with a hyperkinetic apical contraction. In the ECG changes, although ST change followed by $\mathrm{T}$ inversion is a common ECG finding complicated with Takotsubo or reverse Takotsubo cardiomyopathy, left bundle branch block (LBBB) may be a potential ECG pattern which is seen [3]. In addition, LBBB has been considered a mortality predictor in patients with acute coronary artery disease $[4,5]$. To our knowledge, no studies have reported on reverse Takotsubo cardiomyopathy with LBBB after SAH. Therefore, we describe the clinical profile and outcomes of a patient with LBBB and reverse Takotsubo cardiomyopathy during surgery.

\section{Case}

Ethical statements: This study was approved by the Institutional Review Board (IRB) of Yeungnam University Hospital (IRB No: YUMC-2021-08-034), and written informed consent was obtained from the patient.

Received: July 26, 2021 • Revised: September 2, 2021 • Accepted: September 7, 2021

Corresponding author: Eun Kyung Choi, MD, PhD

Department of Anesthesiology and Pain Medicine, Yeungnam University College of Medicine, 170 Hyeonchung-ro, Nam-gu, Daegu 42415, Korea

Tel: +82-53-620-3361•Fax: +82-53-626-5275•E-mail: ekchoe@ynu.ac.kr 


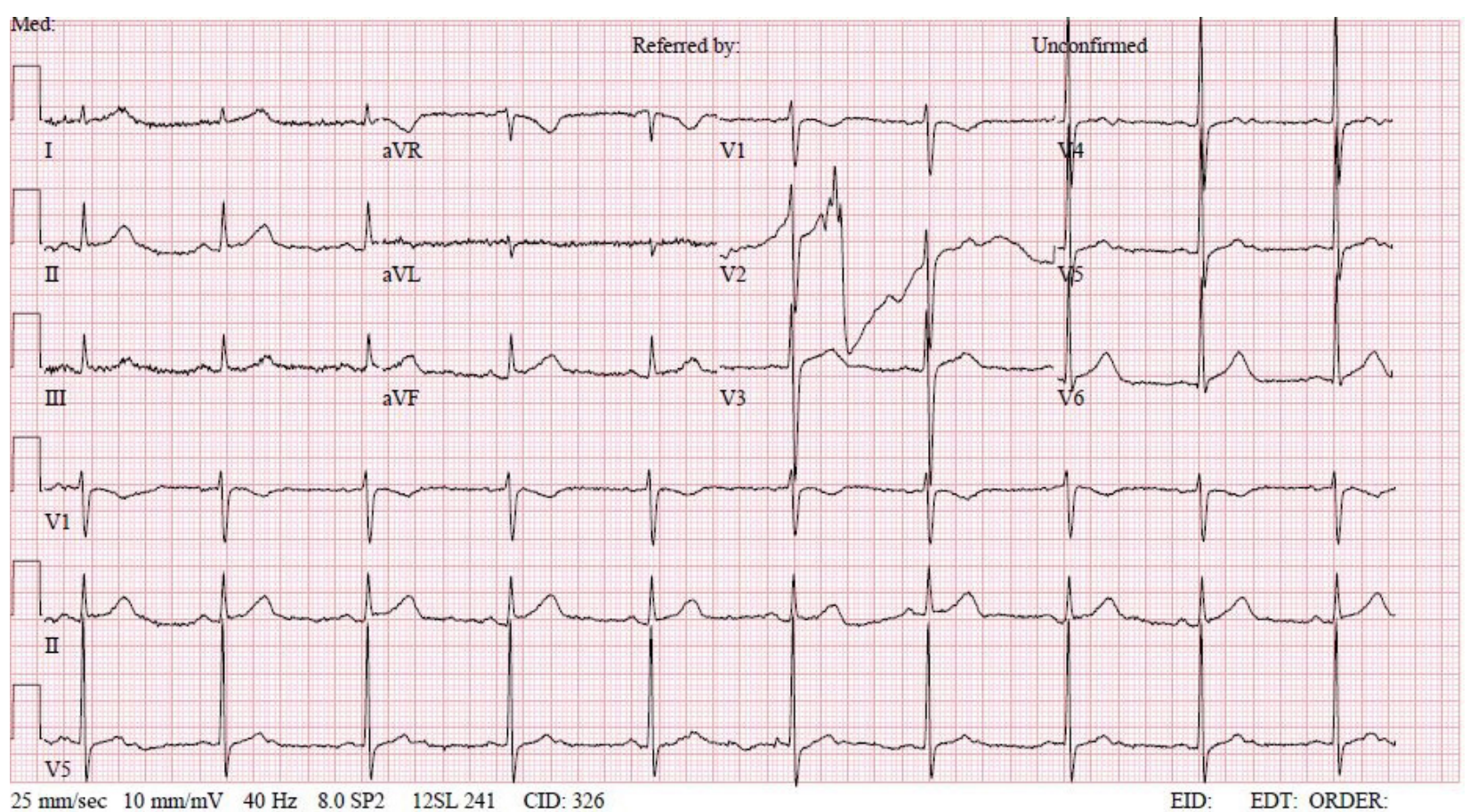

Fig. 1. Preoperative electrocardiogram with normal sinus rhythm.

A 50-year-old male (height, $172 \mathrm{~cm}$; weight, $65 \mathrm{~kg}$ ) with a history of SAH was presented to the emergency department with hydrocephalus after endovascular embolization by a ruptured subarachnoid aneurysm. He was scheduled for urgent external ventricular drainage catheterization. The patient had no cardiac symptoms, and ECG showed a normal sinus rhythm (NSR) (Fig. 1). Troponin-I was measured at $0.05 \mathrm{ng} / \mathrm{mL}$, which was not significantly different from one year previously. Besides this, preoperative laboratory investigations were normal.

In the operating room, the patient's ECG was NSR at 70 beats/ $\mathrm{min}$. General anesthesia was induced with 90 -mg propofol, continuous infusion of remifentanil, and 70-mg rocuronium. Due to insufficient NPO time, tracheal intubation was performed with rapid sequence induction. Immediately after tracheal intubation, the patient showed a blood pressure of $170 / 90 \mathrm{mmHg}$ with a heart rate of 95 beats/min. Anesthesia was maintained with sevoflurane at 1.5 to 2.0 volume $\%$ in $50 \%$ oxygen in air with a continuous infusion of remifentanil ( $0.05-0.1 \mu \mathrm{g} / \mathrm{kg} / \mathrm{min})$. Approximately 10 minutes after tracheal intubation, during the surgeon draping the surgery site for hematoma evacuation and ventricular drainage, the ECG pattern suddenly changed from NSR to LBBB with wide QRS and was noted continuously (Fig. 2). After the operation was halted, a catheter was inserted into the radial artery for continuous hemodynamic monitoring. At that time, portable transthoracic echocardiogram (TTE) showed a depressed left ventricular ejec- tion fraction (LVEF, 36\%) (Fig. 3) while the left ventricular basal and mid segments were severely hypokinetic, while the apical segment was hyperkinetic (Table 1). The right ventricle and valve function were normal. Nonetheless, the patient was not administered hemodynamic support or catecholamine due to stable vital signs; mean arterial pressure $(85-90 \mathrm{mmHg})$ and heart rate $(70$ 80 beats $/ \mathrm{min}$ ) were not significantly different from before ECG changes. Based on the TTE findings and ECG changes, we diagnosed the reverse Takotsubo cardiomyopathy with LBBB. After consultation with an anesthesiologist, neurosurgeon, and caregiver we decided to continue the surgery. Twenty-five minutes after the ECG changes, the patient's ECG returned to NSR immediately after trephination. TTE showed mildly improved LVEF (44\%) (Fig. 4) with mild hypocontractility of the basal segment (Table 1). Postsurgery, the patient was moved to the intensive care unit (ICU) and was intubated to prevent hemodynamic instability.

In the ICU, regional wall motion abnormality at the apical middle area was observed and the LVEF was $40 \%$ at bedside TTE. Troponin-I was then measured at $0.44 \mathrm{ng} / \mathrm{mL}$. On postoperative day 1, a follow-up TTE revealed normal left ventricular wall motion and an LVEF of $63 \%$. The troponin level also decreased steadily, reaching $0.05 \mathrm{ng} / \mathrm{mL}$ on postoperative day 3 . 


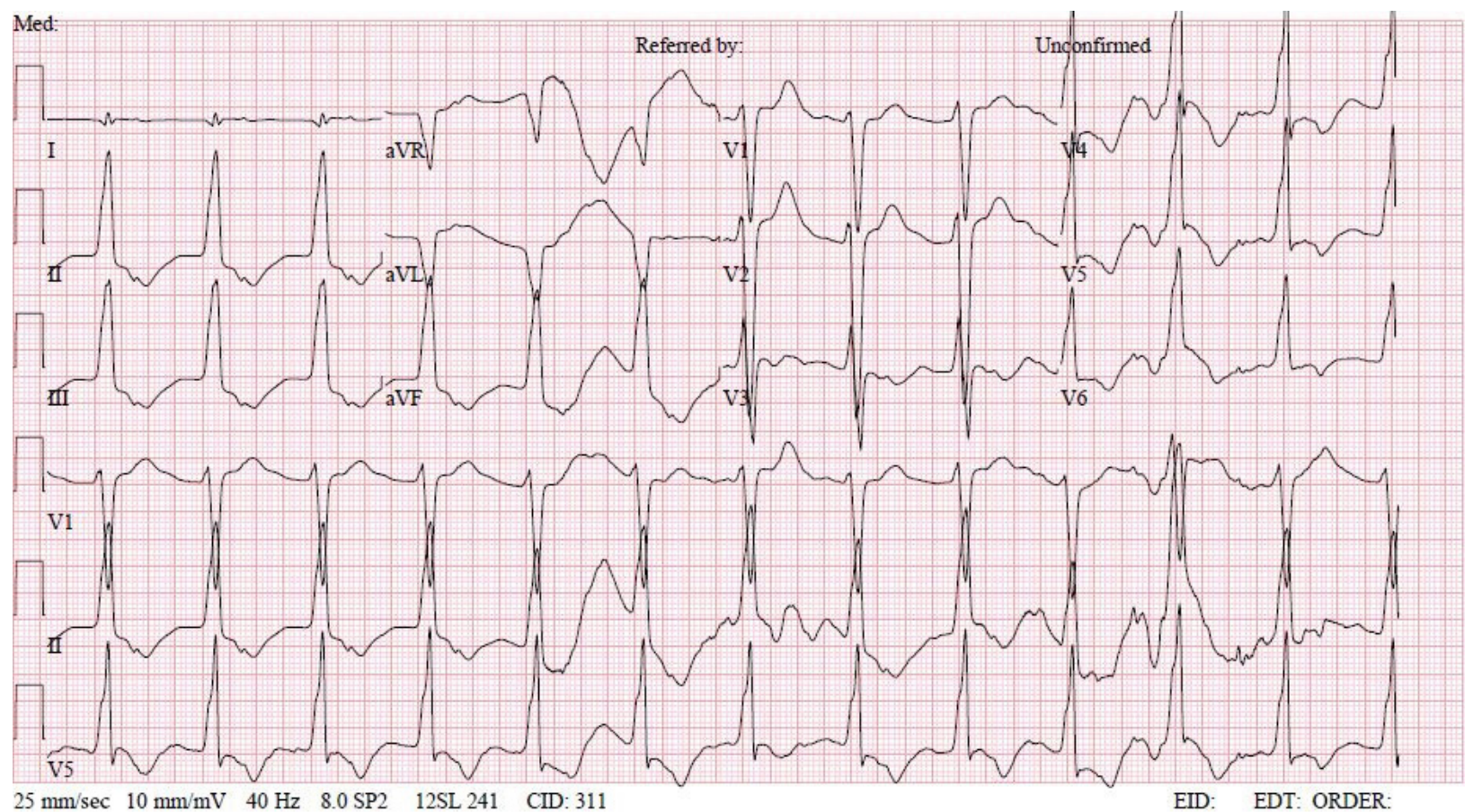

Fig. 2. Intraoperative electrocardiogram with left bundle branch block.

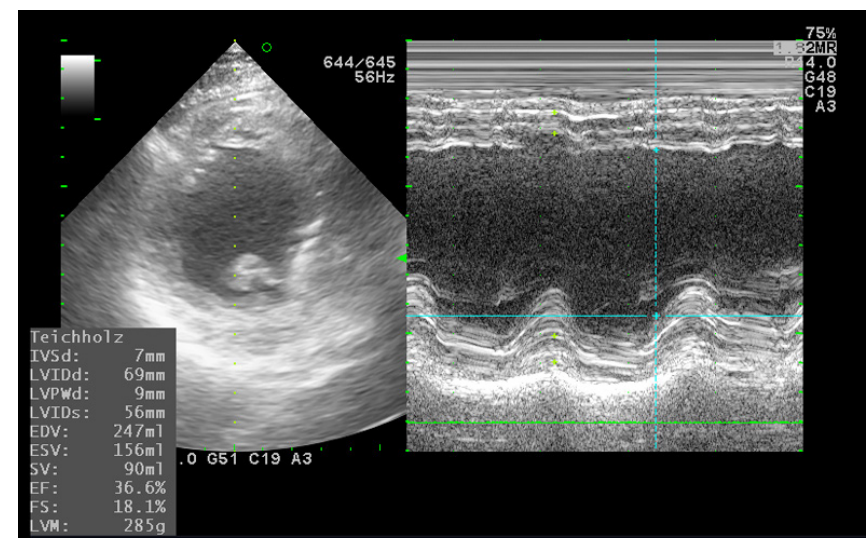

Fig. 3. Intraoperative transthoracic echocardiogram at the left bundle branch block. IVSd, interventricular septum diastolic; LVIDd, left ventricular internal dimension in diastole; LVPWd, left ventricular posterior wall thickness in diastole; LVIDs, left ventricular internal dimension in systole; EDV, end diastolic volume; ESV, end systolic volume; SV, stroke volume; EF, ejection fraction; FS, fractional shortening; LVM, left ventricular mass.

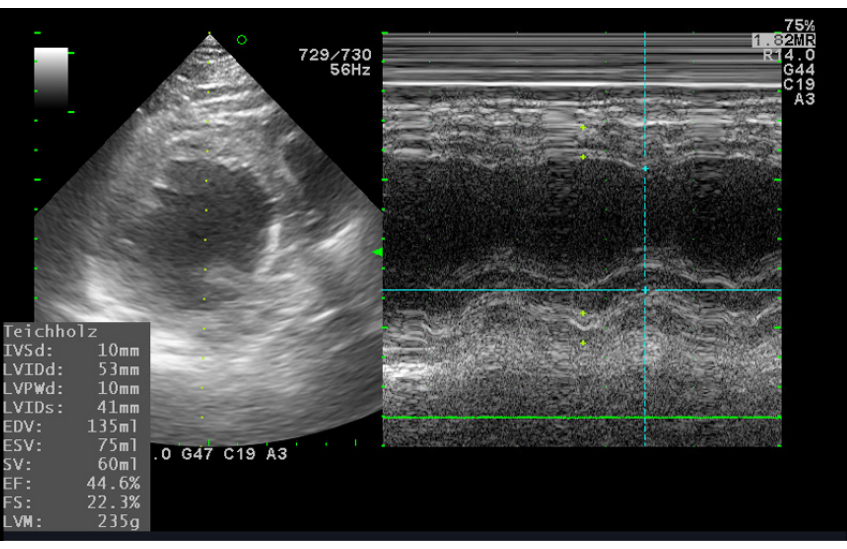

Fig. 4. Intraoperative transthoracic echocardiogram at the normal sinus rhythm. IVSd, interventricular septum diastolic; LVIDd, left ventricular internal dimension in diastole; LVPWd, left ventricular posterior wall thickness in diastole; LVIDs, left ventricular internal dimension in systole; EDV, end diastolic volume; ESV, end systolic volume; SV, stroke volume; EF, ejection fraction; FS, fractional shortening; LVM, left ventricular mass.

Table 1. Transthoracic echocardiogram (TTE) findings

\begin{tabular}{lll}
\hline \multirow{2}{*}{ Variable } & \multicolumn{1}{c}{ Intraoperative TTE } \\
\cline { 2 - 3 } Estimated LVEF & \multicolumn{1}{c}{ Sudden change to LBBB } & \multicolumn{1}{c}{ Convert to NSR } \\
Left ventricular RWMA & Basal and mid segments - severe hypokinetic & Basal segment - mild hypokinetic \\
Valvular state (stenosis/regurgitation) & Apical segment - hyperkinetic & Non-specific \\
\hline
\end{tabular}

LBBB, left bundle branch block; NSR, normal sinus rhythm; LVEF, left ventricular ejection fraction; RWMA, regional wall motion abnormality. 


\section{Discussion}

In this case, we present a patient who developed reverse Takotsubo cardiomyopathy with LBBB after anesthetic induction, which was scheduled as an emergent surgery for brain catheterization after SAH.

Takotsubo cardiomyopathy is a disorder characterized by acute coronary syndrome as follows: ECG change, elevation of cardiac biomarker, and left ventricular wall motion abnormalities not contributing to obstructive coronary disease [1]. More recently, reverse Takotsubo cardiomyopathy has been identified as a variant of Takotsubo cardiomyopathy [2]. The prevalence of reverse Takotsubo cardiomyopathy is lower than that of Takotsubo cardiomyopathy $(2.2 \%$ vs. $82 \%)$ [6]. Takotsubo cardiomyopathy is characterized by transient left ventricular dysfunction with a hypocontractile apex with a compensatory hypercontractile base. While reverse Takotsubo cardiomyopathy shows the opposite left ventricular movement, in other words, a hypokinetic basal ventricular segment with a hyperkinetic apical contraction [2]. The difference in regional wall motion abnormalities between both has been speculated to be the distribution pattern of adrenergic receptors and variations in individual susceptibilities to catecholamine stimulation within the myocardium [7]. This speculation is also supported by differences in age and sex prevalence. In terms of age, adrenoreceptors are more abundant at the base in the younger population, which favors the development of reverse Takotsubo cardiomyopathy [1]. Conversely, Takotsubo cardiomyopathy is more prevalent in postmenopausal age with an abundance of adrenoreceptors at the apex [8]. Moreover, Takotsubo cardiomyopathy has a female predominance [9], while reverse Takotsubo cardiomyopathy showed an increased incidence in men up to $70 \%$ [10].

These clinical syndromes can occur partly due to emotional or physical stress [11]. During the perioperative period, various stresses including anxiety, surgical and anesthetic procedures, and inadequate analgesia can trigger Takotsubo or reverse Takotsubo cardiomyopathy $[12,13]$. Reverse Takotsubo cardiomyopathy is more often associated with triggering stressors than Takotsubo cardiomyopathy $[1,10]$. The catecholamine surge caused by these intense stresses has been considered as the main pathophysiologic mechanism of transient left ventricular dysfunction, which leads to multivessel coronary vasospasm, microvascular dysfunction, and subsequently myocardial stunning with contraction band necrosis [14]. In this case, our patient developed a sudden ECG change (LBBB with wide $\mathrm{QRS}$ ) with left ventricular dysfunction (LVEF, $33 \%$ with hypokinetic basal and mid segments) after anesthetic induction, and returned to NSR ECG with mildly improved left ventricular function (LVEF, 46\%) immediately after ventricular drain- age. We speculate that the patient's physical stress from the induction of anesthesia, such as laryngoscopy, intravenous, and inhalational anesthetics as well as the patient's condition of SAH, as a medical stressor, triggered reverse Takotsubo cardiomyopathy. In addition, decompression of increased intracranial cerebral pressure by trephination may affect the conversion of sudden ECG changes (from LBBB to NSR) with mildly improved left ventricular dysfunction.

Among stress-induced cardiomyopathy, SAH has been well recognized as a priori draw (triggering stressor). Secondary cardiomyopathy has been found in $20 \%$ to $30 \%$ of patients with SAH [15]. High levels of catecholamines in SAH support the main pathophysiology of adrenergic storms, explaining Takotsubo cardiomyopathy or reverse Takotsubo cardiomyopathy. Reverse Takotsubo cardiomyopathy is more common in patients following SAH [16]. The difference of prevalence in patients with SAH seems to be explained by the left ventricle basal segment potentially being more vulnerable to catecholamine toxicity under neurogenic stress as this area has more sympathetic nerve endings, while the apex may be more vulnerable to catecholamine toxicity under general stress as the apex segment has more adrenoreceptors [17]. In a meta-analysis of patients with $\mathrm{SAH}$, stress cardiomyopathy showed poor outcomes and increased mortality [18]; however, myocardial dysfunction is independent of SAH severity [19]. In this case, the patient's condition of SAH was preceded by both surgery and anesthetic stressors, and the patient had no previous history of cardiac disease.

Takotsubo cardiomyopathy is characterized by ischemic ECG changes (either ST-segment elevation and/or T-wave inversion). In some cases, although uncommon, LBBB has been noted as the ECG pattern of Takotsubo cardiomyopathy presentation. Parodi et al. [3] showed a trend towards a poor baseline clinical profile, such as more severe left ventricular systolic dysfunction and delayed left ventricular functional recovery among patients with Takotsubo cardiomyopathy presenting with LBBB. Moreover, on long term follow-up, the patients showed a higher mortality rate than those without LBBB. However, after adjusting for age and baseline properties, LBBB was not found to be an independent predictor of poor outcomes. In reverse Takotsubo cardiomyopathy, the ECG pattern is less understood than that of Takotsubo cardiomyopathy. Elikowski et al. [20] reported that ST segment depression is a more common abnormality; however, ST changes may be absent in the course of reverse Takotsubo cardiomyopathy with intracranial hemorrhage. In our case, we observed ECG changes in the LBBB along with left ventricular dysfunction. To our knowledge, this is the first report of an LBBB pattern in reverse Takotsubo cardiomyopathy. This suggests that any ECG pattern that exhib- 
its evidence of LV dysfunction, as well as the classic form, can be observed in reverse Takotsubo cardiomyopathy. Moreover, LBBB may represent the early ECG phase in reverse Takotsubo cardiomyopathy. Therefore, in case of ECG changes after SAH are incompatible with coronary artery syndrome, Takotsubo cardiomyopathy or reverse Takotsubo cardiomyopathy should be considered as a differential diagnosis. Moreover, as with patients with Takotsubo cardiomyopathy with LBBB, the presence of LBBB in patients with reverse Takotsubo cardiomyopathy may not be an independent predictor of poor outcomes. However, since LBBB has been considered a mortality predictor in patients with acute coronary artery disease $[4,5]$, more cases will need to be evaluated for the assessment of outcomes in reverse Takotsubo cardiomyopathy.

In conclusion, we describe a case of unexpected reverse Takotsubo cardiomyopathy with an LBBB ECG pattern during surgery in a patient with SAH. This case enhances our knowledge of ECG characteristics in reverse Takotsubo cardiomyopathy.

\section{Notes}

\section{Conflicts of interest}

No potential conflict of interest relevant to this article was reported.

\section{Funding}

None.

\section{Author contributions}

Conceptualization, Project administration, Investigation, Supervision: EKC; Data curation, Formal analysis, Resources: MHK; Methodology: EKC, JHK; Visualization: JHK, Writing-original draft: EKC; Writing-review \& editing: JHK.

\section{ORCID}

Eun Kyung Choi, https://orcid.org/0000-0001-5758-6741

Jong-Hoon Kim, https://orcid.org/0000-0001-9492-6901

Minhyun Kim, https://orcid.org/0000-0001-8219-1071

\section{References}

1. Ramaraj R, Movahed MR. Reverse or inverted takotsubo cardiomyopathy (reverse left ventricular apical ballooning syndrome) presents at a younger age compared with the mid or apical variant and is always associated with triggering stress. Congest Heart Fail 2010;16:284-6.

2. Prasad A, Lerman A, Rihal CS. Apical ballooning syndrome
(Tako-Tsubo or stress cardiomyopathy): a mimic of acute myocardial infarction. Am Heart J 2008;155:408-17.

3. Parodi G, Salvadori C, Del Pace S, Bellandi B, Carrabba N, Gensini GF, et al. Left bundle branch block as an electrocardiographic pattern at presentation of patients with Tako-tsubo cardiomyopathy. J Cardiovasc Med (Hagerstown) 2009;10:100-3.

4. Godman MJ, Lassers BW, Julian DG. Complete bundle-branch block complicating acute myocardial infarction. N Engl J Med 1970;282:237-40.

5. Brilakis ES, Wright RS, Kopecky SL, Reeder GS, Williams BA, Miller WL. Bundle branch block as a predictor of long-term survival after acute myocardial infarction. Am J Cardiol 2001; 88:205-9.

6. Templin C, Ghadri JR, Diekmann J, Napp LC, Bataiosu DR, Jaguszewski M, et al. Clinical features and outcomes of Takotsubo (stress) cardiomyopathy. N Engl J Med 2015;373:929-38.

7. Mori H, Ishikawa S, Kojima S, Hayashi J, Watanabe Y, Hoffman $\mathrm{JI}$, et al. Increased responsiveness of left ventricular apical myocardium to adrenergic stimuli. Cardiovasc Res 1993;27:192-8.

8. Ramaraj R. Stress cardiomyopathy: aetiology and management. Postgrad Med J 2007;83:543-6.

9. Ramaraj R, Sorrell VL, Movahed MR. Levels of troponin release can aid in the early exclusion of stress-induced (takotsubo) cardiomyopathy. Exp Clin Cardiol 2009;14:6-8.

10. Song BG, Chun WJ, Park YH, Kang GH, Oh J, Lee SC, et al. The clinical characteristics, laboratory parameters, electrocardiographic, and echocardiographic findings of reverse or inverted takotsubo cardiomyopathy: comparison with mid or apical variant. Clin Cardiol 2011;34:693-9.

11. Roshanzamir S, Showkathali R. Takotsubo cardiomyopathy a short review. Curr Cardiol Rev 2013;9:191-6.

12. Yoshikawa T. Takotsubo cardiomyopathy, a new concept of cardiomyopathy: clinical features and pathophysiology. Int J Cardiol 2015; 182:297-303.

13. Liu S, Bravo-Fernandez C, Riedl C, Antapli M, Dhamee MS. Anesthetic management of Takotsubo cardiomyopathy: general versus regional anesthesia. J Cardiothorac Vasc Anesth 2008; 22:438-41.

14. Lindsay J, Paixao A, Chao T, Pichard AD. Pathogenesis of the Takotsubo syndrome: a unifying hypothesis. Am J Cardiol 2010;106:1360-3.

15. Bybee KA, Prasad A. Stress-related cardiomyopathy syndromes. Circulation 2008;118:397-409.

16. Hravnak M, Frangiskakis JM, Crago EA, Chang Y, Tanabe M, Gorcsan J 3rd, et al. Elevated cardiac troponin I and relationship to persistence of electrocardiographic and echocardiographic abnormalities after aneurysmal subarachnoid hemorrhage. 
Stroke 2009;40:3478-84.

17. Kristensen SL, Mogensen UM, Jhund PS, Petrie MC, Preiss D, Win S, et al. Clinical and echocardiographic characteristics and cardiovascular outcomes according to diabetes status in patients with heart failure and preserved ejection fraction: a report from the I-Preserve Trial (Irbesartan in Heart Failure with Preserved Ejection Fraction). Circulation 2017;135:724-35.

18. van der Bilt IA, Hasan D, Vandertop WP, Wilde AA, Algra A, Visser FC, et al. Impact of cardiac complications on outcome after aneurysmal subarachnoid hemorrhage: a meta-analysis.
Neurology 2009; 72:635-42.

19. Schuiling WJ, Dennesen PJ, Tans JT, Kingma LM, Algra A, Rinkel GJ. Troponin I in predicting cardiac or pulmonary complications and outcome in subarachnoid haemorrhage. J Neurol Neurosurg Psychiatry 2005;76:1565-9.

20. Elikowski W, Małek-Elikowska M, Kudliński B, Skrzywanek P, Smól S, Rzymski S. ECG pattern in reverse takotsubo cardiomyopathy demonstrated in 5 cases with intracranial hemorrhage. Pol Merkur Lekarski 2016;41:136-40. 